\title{
Developing a co-produced e-learning programme to support marginalised medically underserved patients
}

$\mathrm{PJ}$ pharmaceutical-journal.com/news-and-analysis/opinion/correspondence/developing-a-co-produced-e-

The Pharmaceutical Journal 26 NOV

2019

There have been recent calls for the NHS to pursue a value-based healthcare system, one that is "equitable, sustainable and transparent" $[1]$. However, promoting a culture based on the values of patient-centred care can be challenging. One notable example in pharmacy was the concern that medicines use reviews (MURs) were being driven by organisational targets in preference to patient need[2]. Consequently, in 2019, it was announced that MURs would be phased out and replaced by structured medication reviews, which will be carried out by pharmacists working within primary care networks[3].

Vulnerable people belonging to marginalised groups (e.g. those with disabilities, who are homeless, or from the black, Asian and minority ethnic [BAME] community) often experience inequitable access to healthcare, poorer patient-professional communication and sometimes racism or cultural bias $[4],[5]$. They may hold strong beliefs that they cannot be helped, face discrimination or disempowerment because of their circumstance and are often disenfranchised from mainstream primary healthcare services $[6],[7],[8]$. Inequitable access to routine and preventative care increases demand on acute services, contributes to emergency admissions and adds further pressure to an already stretched healthcare system $[9]$.

Recent research has indicated that marginalised or medically "underserved" patients are not accessing or being offered medicine support services, including MURs $[10]$. To redress the balance and to raise awareness of this group of patients, an e-learning resource for pharmacy staff was developed using a co-production approach. Co-production is a model that actively involves service users, carers and communities in equal partnership through acknowledging patients' "lived experience". They are best placed to advise on the support and services that will make a positive difference to them[11].

In total, 57 participants (33 patients and 24 pharmacy professionals) attended one of four half-day co-production workshops in Nottingham during autumn and winter 2016. Patient representation was from a range of marginalised groups, including the asylum and refugee community; the BAME community; those with disabilities; those who were homeless; those who were housebound; the lesbian, gay, bisexual and transgender and queer community; those diagnosed with mental illness; people from the Gypsy, Roma and Traveller communities; and older people with multiple morbidity and medicines.

Pharmacy representation was from community pharmacy staff (i.e. pharmacists, accredited technicians, dispensers, medicine counter assistants); superintendent pharmacists; community pharmacy owners; and pharmacy representatives, professional leadership and educational bodies (i.e. local pharmaceutical committees, the Royal Pharmaceutical Society, the Centre for Pharmacy Postgraduate Education). 
To ensure the widest possible patient participation and representation, tried and tested engagement strategies were deployed[12]. These included using extended recruitment and engagement time frames, planning for higher resourcing costs and operating via community partnership. A $£ 200$ inconvenience allowance was made available to all workshop participants, whether they were patients or pharmacy staff. To support and further the findings from the workshops, ten one-to-one interviews were also conducted with patients from marginalised groups.

At these workshops, discussions centred around medicines issues and the challenges faced when using pharmacies and the wider healthcare system. The contents of the elearning programme were incrementally built and refined over the four workshops. Personal written reports, along with group suggestions to improve the MUR service, were captured on storyboards to elicit a more detailed plan for the contents of the learning. The final specification was then passed on to the Health e-Learning and Media team at the University of Nottingham, which used this information to create the digital resource.

Broadly, the findings supported previous literature that many patients experienced significant problems with access to healthcare, problems with medicines (i.e. nonadherence), and that their status or circumstances often left them feeling socially isolated. When asked about their experiences of pharmacy, opinions were mixed, with some patients expressing gratitude when they found this to be a friendly and useful encounter. However, others perceived pharmacies as busy places and this limited the scope for patients to see them as a places to access or resolve health or medicinerelated issues.

The final co-produced resource consists of the following three components:

1. Discovering and understanding under-served communities;

2. Exploring the medicine experiences and needs of patients who are under-served;

3. Effectively interacting and engaging patients who are under-served.

The co-produced digital educational resource can be freely accessed and shared with the pharmacy team through this link.

Since the development of the co-produced e-learning resource, its impact and effectiveness have been tested in a before and after comparison evaluation study 113$]$. The resource was effective at engaging with and improving the perceived capabilities of pharmacy professionals towards groups that are medically under-served, however, the findings also indicated that organisational barriers may hinder the translation of learning into effective behaviour change $\frac{[14]}{}$.

Improving fairness, social justice and addressing inequitable access to health services features as a priority for the NHS[15]. Employing the co-production approach has the potential to transform the delivery of pharmacy services, reduce healthcare inequity and promote value-based healthcare. Our work shows that this process can guide reconfiguration of a service and lead to care being provided to those most in need.

Asam Latif, senior research fellow, University of Nottingham

Baguiasri Mandane, pharmacist, Guy's and St Thomas' NHS Trust

Nargis Gulzar, senior lecturer, Leicester School of Pharmacy, De Montfort University

Citation: The Pharmaceutical Journal, online, online | DOI: 10.1211/PJ.2019.20207364 
- Previous

- Next
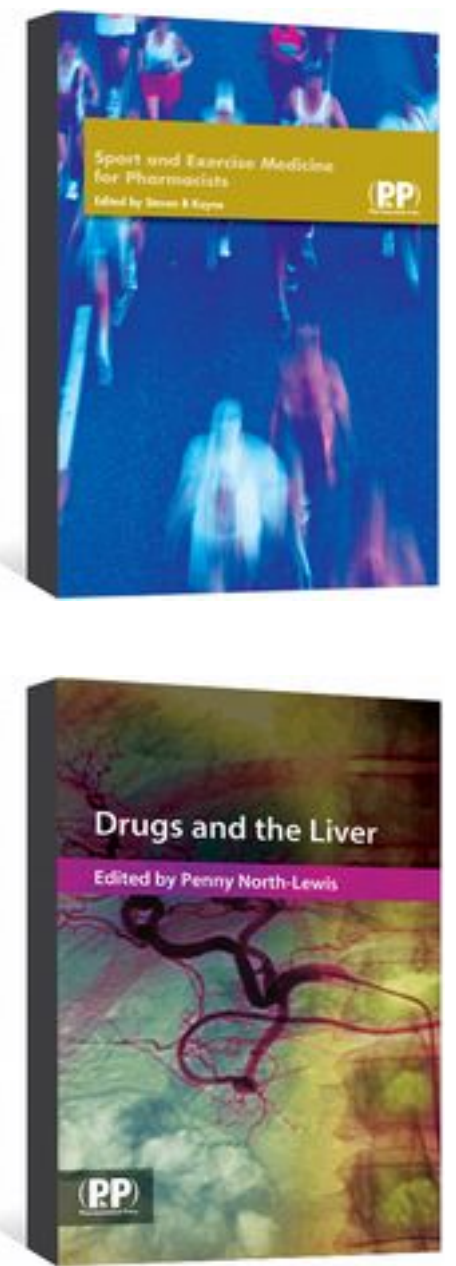

- Drugs and the Liver

Drugs and the Liver assists practitioners in making pragmatic choices for their patients. It enables you to assess liver function and covers the principles of drug use in liver disease.

\section{$£ 38.00$ Buy now}




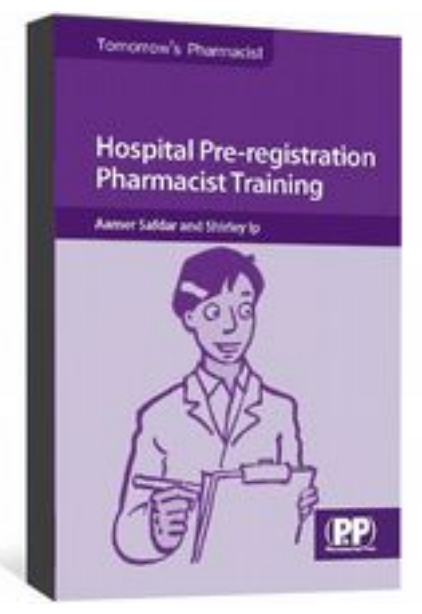

\section{- Hospital Pre-registration Pharmacist Training}

A practical explanation for undergraduates and pre-registration trainees. Shows what to expect from a hospital pre-registration pharmacist training programme.

\section{$£ 25.00$ Buy now}

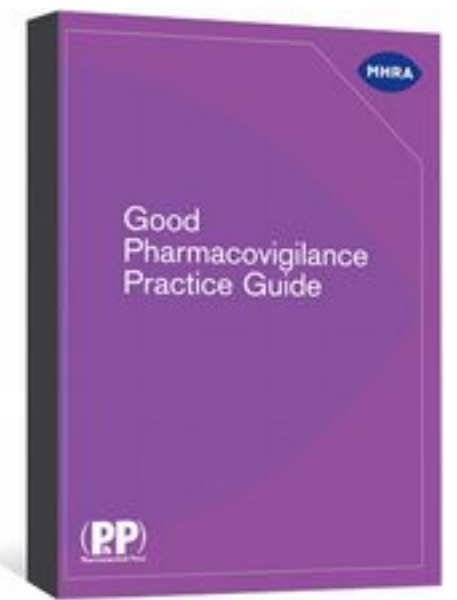

\section{- Good Pharmacovigilance Practice Guide}

An essential guide on pharmacovigilance of medicinal products for human use. Practical advice for developing effective pharmacovigilance systems.

£38.00Buy now 


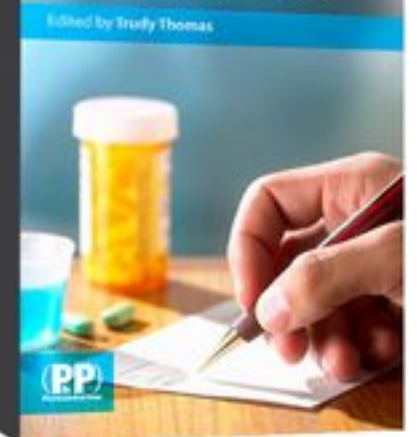

- Developing Your Prescribing Skills

Developing Your Prescribing Skills uses case studies, mind maps and feedback from experienced prescribers. It supplies practical advice on the issues facing prescribers in all types of practice.

£23.00Buy now

Search an extensive range of the world's most trusted resources

Powered by MedicinesComplete 